\title{
INDUSTRIAL APPLICATIONS OF PHOTOCHEMISTRY
}

\author{
MARTIN PAPE \\ BASF AG, Hauptlaboratorium, 67 Ludwigshafen, GFR
}

\begin{abstract}
Starting from the various primary photochemical procésses (luminescence, non-radiative transition to the ground state, electron and energy transfer, isomerization, addition, hydrogen abstraction, and fragmentation), the most important technical applications of photochemistry are reviewed. The main applications of luminescence phenomena are optical bleaching of textiles and paper. Rapid radiationless transitions to the ground state, often brought about by means of quenchers or of thermoreversible light reactions, are necessary for light protection of plastics and human skin. Electron and energy transfer processes have found a wide range of applications in photography, preparative photochemistry, and light sources, respectively. Photofragmentations are used in reprography and in the photochemical synthesis of detergents, insecticides and monomers for polyamides.
\end{abstract}

For the industrial chemist, photochemistry is just one of the many means of producing chemical compounds or bringing them into reaction. However, it has some advantages over thermal, catalytic and other methods that immediately fascinate him. These include:

(1) Selective activation of individual reactants,

(2) Specific reactivity of electronically excited molecules,

(3) Low thermal load on the reaction system,

(4) Exact control of radiation in terms of space, time and energy.

But photochemistry is not without its own specific problems, including:

(1) Absorption characteristics-Only absorbed light can be exploited for chemical purposes. For this reason, many reaction systems are ruled out for photoreactions because of their unfavourable absorption characteristics.

(2) Internal and external light filters-Photoreactions may be rapidly terminated if products with competing absorptions are formed.

(3) Investment costs-Photochemical production plants may incur high unit capital costs if the space-time yield is low as a result of limitations imposed by the power of the lamps.

(4) Electricity costs-Light is more expensive than heat because considerable losses occur in the production of electrical energy and its conversion into usable light energy. 


\section{MARTIN PAPE}

With these points in mind, the industrial photochemist concentrates on reaction systems that bring out the full advantages of photochemistry and minimize the difficulties inherent in the method.

The following objects have proved to be of economic interest:

(1) Use of light for synthesis,

(2) Synthesis of photosensitive compounds,

(3) Development of u.v.-stabilizers,

(4) Synthesis of compounds with specific spectral properties,

(5) Contributions to ecology.

The main aim of preparative photochemistry is to reduce manufacturing costs for chemical products by introducing photochemical steps in the syntheses.

Light-sensitive compounds have great technical significance in photography, reprography, and printing. Important applications have been also found in u.v.-curable paints, primers, and printing inks.

Photostabilizers are primarily used in plastics and man-made fibres. Another interesting field of application is sunscreen cosmetics. In the synthesis of compounds with special spectral properties, attention is focused on lightfast dyes, optical brighteners, fluorescent dyes, and chemiluminescent systems.

The main photochemical contributions to ecology are chemical storage of solar energy, investigations on the photodegradation of biologically active ingredients, the investigation of photochemical smog formation, and the development of photodegradable plastics.

For all these tasks the theoretical and practical assistance of pure photochemists is extremely welcome. Difficulties for the joint discussion may sometimes arise due to the fact that the photochemists at the universities are usually less concerned about commercial products and their markets than about reactions and mechanisms.

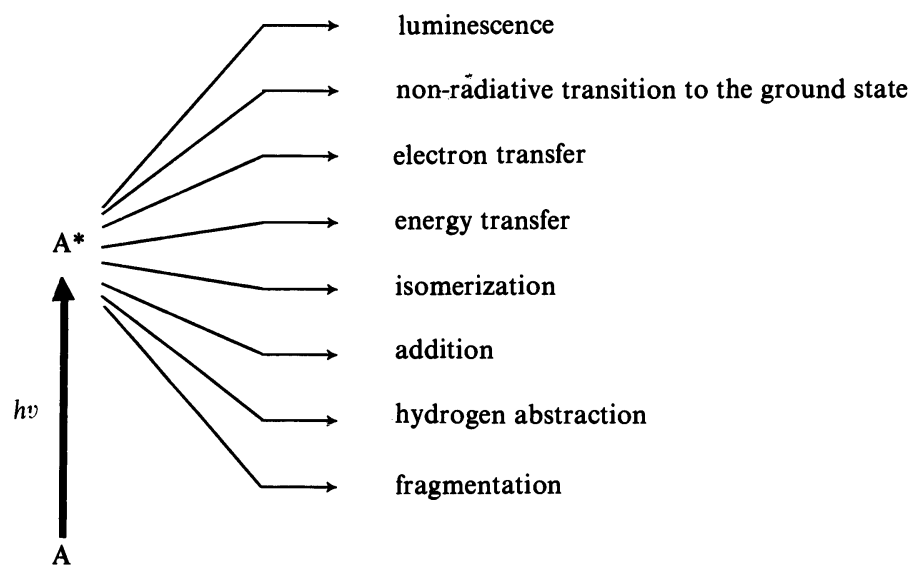

Figure 1. Excited state reaction paths. 


\section{INDUSTRIAL APPLICATIONS OF PHOTOCHEMISTRY}

However, a close connection can hardly be recognized between the type of reaction and the technical application. Thus various mechanisms, e.g. luminescence, energy transfer, non-radiative deactivation, or thermally reversible isomerization are used for photostabilization. Likewise, the initiator radicals in photopolymerization and photocrosslinking reactions may be produced, e.g. by hydrogen abstraction or by fragmentation.

The reason for this diversity is that optimization of technical products and systems often has to meet many mutually conflicting demands. An ultra-violet stabilizer may incorporate the most advanced scientific knowhow, but it would not sell if, say, it were too sparingly soluble in the polymer, if it were too volatile or readily extractable, if it did not have sufficient resistance to heat and chemicals to withstand processing of the plastics, or if it did not appear to be entirely physiologically harmless. Conversely, a stabilizer may be third-rate according to photochemical criteria but could turn out to be a tremendous sales success, simply because it is particularly cheap to produce.

Coping with so many parameters is a great challenge to the technical knowledge and imagination of the industrial photochemist. He must recognize all possible economic advantages and opportunities of photochemical reactions and then put them into practice. Up-to-date knowledge of technology is essential. Thus I shall begin my review with the primary processes starting from the electronically excited states and then introduce you to their most important technical applications realized so far. I have chosen this approach to attract also the interest of pure photochemists because I am sure that with their aid and cooperation we shall be in a better position to find further practical applications of photochemistry.

\section{LUMINESCENCE}

A primary photochemical process of great theoretical and practical signiffcance is luminescence (Figure 2). The electronically excited states responsible for fluorescence and phosphorescence are usually generated by light absorption. But in addition chemiluminescent systems are gaining increasing attention.

An instructive example for the technical exploitation of fluorescence phenomena is the development of optical brighteners ${ }^{1}$. Since the impression made by a white colour is associated with the terms cleanliness and purity, the degree of whiteness of many commercial products, particularly paper and household linen, has become a symbol for quality. Just think of the advertising slogan, 'whiter than white'.

An optimum impression of whiteness is obtained when a body reflects all visible light. However, many materials cannot satisfy this condition, because they absorb some light in the blue range of the spectrum. This leads to what is called the 'blue defect' in the light reflected, and the materials assume a pale yellowish coloration. Nevertheless, the impression of whiteness can be achieved by adding blue fluorescent compounds, which absorb invisible ultra-violet radiation and transform it into visible blue fluorescent light. Figure 3 shows how optical brighteners work. Curve No. 1 is the reflection curve for an unbleached fabric. The blue defect is proportional to the area between curve No. 1 and the straight line for 100 per cent reflection. 
MARTIN PAPE

Type of reaction

Examples

Technical applications

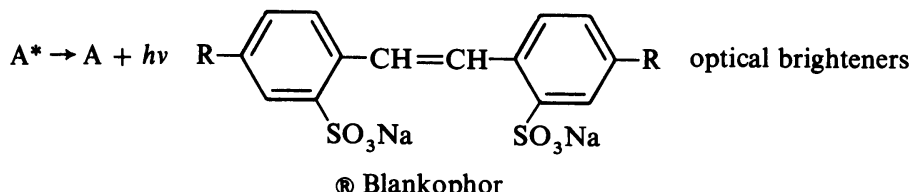

(B) Blankophor<smiles>Oc1ccccc1/C=N/N=C/c1ccccc1O</smiles>

fluorescent dyes

(B) Lumogen UV<smiles></smiles>

chemiluminescent light sources

(B) Coolite

Figure 2. Luminescence

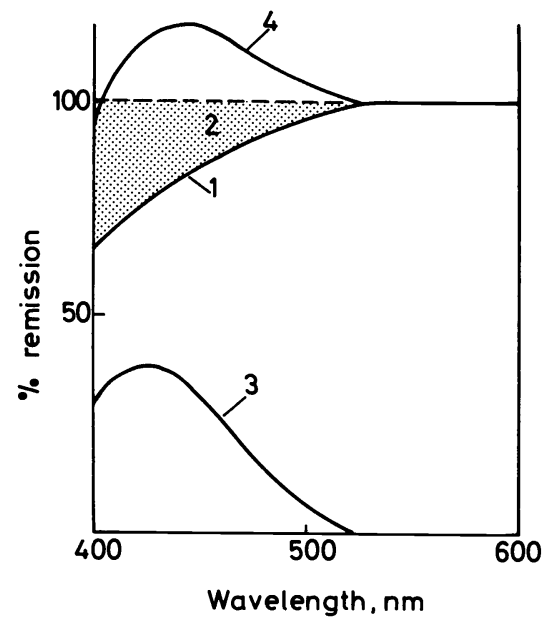

Figure 3. How optical brighteners work: 1 Remission of unbleached fabric, 2 Blue defect, 3 Emission of optical brightener, 4 Remission of unbleached fabric + brightener. 


\section{INDUSTRIAL APPLICATIONS OF PHOTOCHEMISTRY}

Curve No. 3 is the emission of the optical bleach, and curve No. 4 is the sum of curves 1 and 3, i.e. the light reflected by the bleached fabric. The result is a desired slightly bluish white colour. It is 'whiter than white', because more visible light is reflected than is incident.<smiles>[R]c1nc([R])nc(Nc2ccc(/C=C/c3ccc(Nc4nc([R])nc([R])n4)cc3S(N)(=O)=O)c(S(C)(=O)=O)c2)n1</smiles><smiles>[R2][C@@H](COO)NCc1ccc(NCCOO)cc1</smiles><smiles>[R]O[R16](=O)(O)O[Na]</smiles><smiles>[R]c1ccc2oc(/C=C/c3nc4ccc([R])cc4o3)nc2c1</smiles>

Figure 4. Most important commercial brighteners and their technical applications.

One feature of all optical brighteners is their rigid and flat molecular structure, which favours high fluorescence yields. Furthermore they should allow modification by a wide variety of substituents, in order to accommodate different substrates. The current annual production of optical brighteners is about $65000-70000$ tons with a value of about $\$ 350$ million. More than half is consumed by the detergents industry, and one-quarter is used for optical bleaching of paper.

Fluorescent dyes are entirely analogous to optical bleaches. Here again, short-wave light is converted into long-wave fluorescent light in the visible spectrum. These dyes are encountered-as luminous colours in advertising, shock colours in fashion, and warning colours in traffic. As a result of the addition of reflection and fluorescence, fluorescent dyes often emit twice as much light at the longer wavelengths as is contained in incident daylight. 


\section{MARTIN PAPE}

Green, blue and red fluorescent pigments had to be developed for colour television. In this case, however, the excitation is caused by cathode rays and not by ultra-violet light. Fluorescent or phosphorescent stamps are used for automatic sorting of letters and postcards. Another lucrative application for phosphorescent or luminous paints is marking dials and light switches.

The avant-garde of the commercial chemiluminescent products has also started to secure a foothold on the market. An example is American Cyanamid's ${ }^{\circledR}$ Coolite $^{2}$. Its main application is emergency lighting in mines, factories and camping sites. Steps have already been taken to equip lifeboats with a substance that lights up when it comes into contact with water and thus surrounds the boat with an illuminated zone that can be seen from afar at night.

\section{NON-RADIATIVE DEACTIVATION}

The effects of luminescence literally hit the eye. Conversion of the electronic energy into heat is less striking. Nevertheless, non-radiative deactivation to the ground state has a much more far-reaching significance. Almost all

Type of reaction: $A^{*} \rightarrow A+\Delta H$

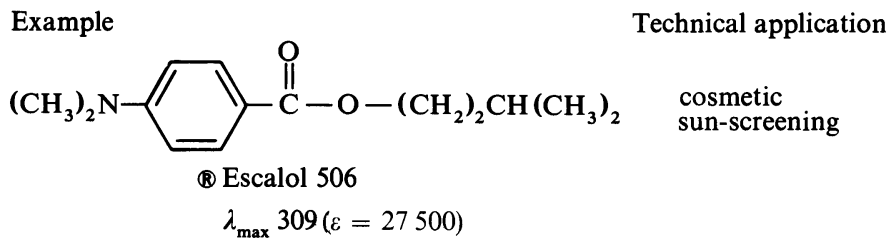

Figure 5. Non-radiative deactivation.

objects that we encounter in everyday life, such as foodstuffs, plastics, dyes or textiles, are changed on exposure to light and are finally destroyed. It is thus hardly surprising that more chemists are working at stopping and alleviating the damaging effects of light than are looking for new means of exploiting light. The market in sunscreen cosmetics, which is estimated at more than $\$ 40$ million in West Germany alone, demonstrates how profitable this task can be.

The function of a sunscreen cosmetic is to filter solar radiation ${ }^{3}$. The rays that are most responsible for erythema, i.e. those between 290 and $320 \mathrm{~nm}$, are filtered out, and those that tan direct, i.e. those between 320 and $390 \mathrm{~nm}$, are allowed to pass through unhindered. Thus an attractive tan is obtained without sunburn.

Up till now, esters of $p$-dialkylaminobenzoic acid have proved to be very effective sunscreens. They display intense ultra-violet absorption at about $308 \mathrm{~nm}$ and almost complete light transmittance in the range of frequencies in which the skin is pigmented. They fulfil further requirements in being highly resistant to light, heat, oxygen, water and sweat. Moreover, they are nontoxic and odourless.

Solutions of most sunscreens fluoresce to some extent or other. After they have been applied to the skin, part of the absorbed radiation is not converted 


\section{INDUSTRIAL APPLICATIONS OF PHOTOCHEMISTRY}

to heat but is re-emitted as fluorescent light. However, the formation of singlet states that are capable of fluorescence is associated with the risk of imparting the electronic excitation energy to the adjacent skin. For this reason, it would be interesting to synthesize photostable compounds that are capable of converting absorbed light into heat with a high degree of efficiency.

\section{ELECTRON TRANSFER}

Whereas non-radiative deactivation gives rise to particularly light-stable compoufids, photochemically induced electron transfer is exploited for the development of systems that are extremely sensitive to light. There are

Type of reaction

$$
\mathrm{A}^{*} \rightarrow \mathrm{A}^{+}+\mathrm{e}^{-}
$$

Examples

$$
\begin{aligned}
(\mathrm{AgBr})_{n}^{*} & \rightarrow(\mathrm{AgBr})_{n}^{+}+\mathrm{e}^{-} & & \text {photography, phototropic glasses } \\
\text { dye }^{*} & \rightarrow \text { dye }^{+}+\mathrm{e}^{-} & & \text {spectral sensitization } \\
(\mathrm{ZnO})_{n}^{*} & \rightarrow(\mathrm{ZnO})_{n}^{+}+\mathrm{e}^{-} & & \text {electrophotography }
\end{aligned}
$$

Figure 6. Electron transfer.

innumerable applications for photoconductors in photography, reprography and electronics. Their mode of action is best illustrated by the energy band model.

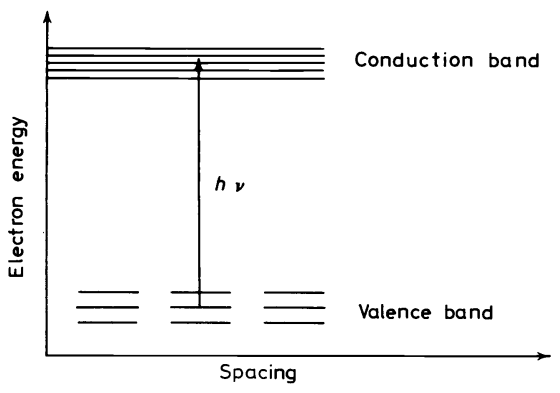

Figure 7. Energy band diagram for photo-conductors.

According to this model, there are two groups of energy levels for the electrons in the crystal lattice, viz. the valence band with its localized electrons and the conduction band, in which the electrons are free to move. As a result of the absorption of light quanta in the crystal, electrons are raised from the valence band into the conduction band and can now be used for reduction processes or for carrying current.

Thus classical silver salt photography is based on the reducing action of the photochemically liberated electrons. They combine with silver ions located at lattice dislocations to form silver atoms. As a result, silver nuclei are formed inside the silver halide crystals and on their surfaces. On subsequent develop- 
ment with a reducing agent, the reduction proceeds only at those crystals at which silver nuclei already exist; and the more silver nuclei present, the faster the development proceeds. By this means, the primary photochemical effect

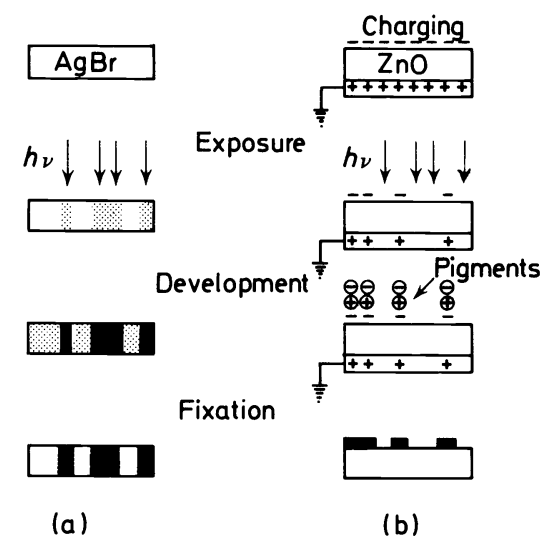

Figure 8. Processes in silver halide photography (a) and in electrophotography (b).

is increased by a factor of $10^{6}$ to $10^{8}$. The electrofax process is illustrated in Figure 8(b). A sheet of paper impregnated with zinc oxide is subjected to a corona discharge. Thus a potential difference between paper and the supporting metal base is generated. On irradiation, electrons in the $\mathrm{ZnO}$ particles are mobilized, and the charges are neutralized. Since this process is restricted to the irradiated areas, a latent electrostatic image is obtained that is made visible by treatment with charged pigments. Even more important is the related Xerox process. Here the image is formed on a photoconductive selenium coating from which it is transferred to ordinary paper.

This technique is unsuitable for multi-coloured prints, which, however, have recently been made possible by photoelectrophoresis and are based on the spectrally selective migration of dispersed pigment particles to produce the colour image ${ }^{4}$.

\section{ENERGY TRANSFER}

A very important contribution made by photography towards the advance of photochemistry in general was the discovery of the phenomenon of photosensitization in 1873. By adsorbing certain organic dyes on silver halide crystallites, their spectral sensitivity is increased to longer wavelengths well beyond the limits imposed by the crystallites' own absorption ( $c a .520 \mathrm{~nm}$ in the case of $\mathrm{AgBr}$ ). The development of panchromatic films and colour photography has been based on this effect. Recent experiments indicate that sensitization is caused by electron transfer from the electronically excited dye molecule to the silver halide lattice. As opposed to crystals, discrete 
atoms and molecules are most often sensitized by transfer of electronic excitation energy rather than by transfer of electrons.

Type of reaction

Examples

Technical applications

$$
\mathrm{A}^{*} \stackrel{+\mathrm{B}}{\rightarrow} \mathrm{A}+\mathrm{B}^{*}
$$

$$
\begin{aligned}
& \mathrm{Hg}^{*}+\mathrm{Tl} \rightarrow \mathrm{Hg}+\mathrm{Tl}^{*} \\
& \text { rose bengal }{ }^{*}+{ }^{3} \mathrm{O}_{2} \rightarrow \\
& \text { rose bengal }+{ }^{1} \mathrm{O}_{2} \\
& \text { polymer* }+ \text { Ni-chelate } \rightarrow \\
& \text { polymer }+ \text { Ni-chelate }+ \text { heat }
\end{aligned}
$$$$
\text { doped mercury lamps }
$$

photooxidation by singlet oxygen

photostabilization of plastics by quenchers

Figure 9. Energy transfer.

An economically interesting application for energy transfer in the gas phase is modern high-wattage lamps for illuminating streets, sporting events and greenhouses. Volatile metal compounds are added in mercury vapour lamps with the aim of making the emission spectrum similar to that of the solar spectrum. These compounds accept the excitation energy of the mercury atoms, and emit light in the desired range of wavelengths.

The principle of energy transfer can be illustrated by a simplified term diagram. The example taken is of great technical importance, viz. doping with thallium iodide.

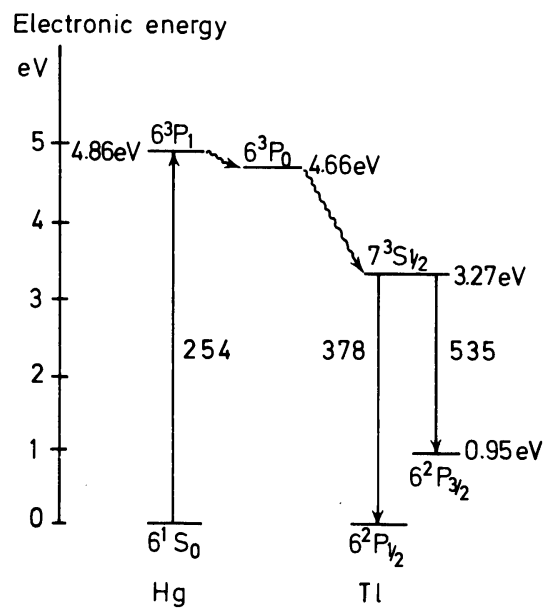

Figure 10. Hg-sensitized luminescence of $\mathrm{Tl}$.

As a result of excitation and collisions of the second kind, the mercury atoms populate a metastable triplet state with a lifetime of up to $10^{-2}$ second. Thus they have sufficient time to collide with and transfer their energy to the thallium atoms, which are present in a low concentration. The thallium atoms, in turn, emit at $535 \mathrm{~nm}$ and $378 \mathrm{~nm}$. The next diagram compares the emission spectrum of an undoped with that of a thallium-doped $40-\mathrm{kW}$ 

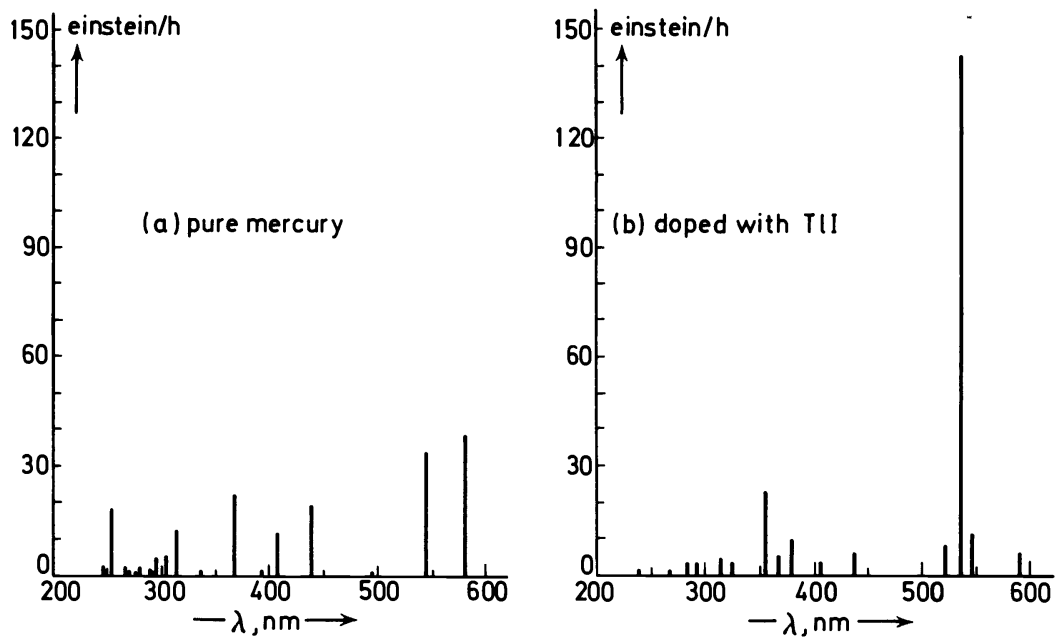

Figure 11. Emission spectra of $40 \mathrm{~kW}$ high pressure mercury lamps.

high-pressure mercury vapour lamp 5 . In the emission spectrum for the thallium iodide doped lamp, you can see the drastic reduction in the mercury lines and the appearance of the characteristic thallium lines, the most intense of which occurs at $535 \mathrm{~nm}$. The doping technique has now been so refined that carefully engineered mixtures of various metals can be added to the mercury discharge without detracting from the beneficial properties of the mercury vapour lamp, viz. high energy density, good efficiency in terms of electricity consumption, and a long life time.

This successful development has directly benefited photochemistry. A crucial factor in the economics of many photoreactions is the availability of lamps that emit the maximum possible amount of effective light quanta for a given electricity consumption. Hence it is now part of the programme for the technical optimization of photoreactions to measure how the quantum yield depends on wavelength and to adjust the light source accordingly. Thus one of the breakthroughs in the technical realization of the photooximation of cyclohexane was made possible by halving the electricity consumption per ton of cyclohexanone oxime by adding $0.07 \mathrm{mg} \mathrm{Tl} / \mathrm{cm}^{3}$ to the mercury vapour lamp ${ }^{6}$.

In many cases, spectral adjustment of the lamps is supplemented by sensitization of the reaction system. Singlet oxygen photochemically generated by triplet energy transfer can enter into a number of reactions that ground state oxygen is incapable of. A well known example is the synthesis of ascaridole discovered by G. O. Schenck ${ }^{7}$. After 1945 this diene reaction of $\alpha$-terpinene with singlet oxygen was carried out on a technical scale. At that time, ascaridole had some significance as an anthelmintic, and previously it could only be obtained from natural oil of chenopodium, Figure 12.

The ene-reaction with oxygen is being used by two perfumery manufacturers, Dragoco (West Germany) and Firmenich (Switzerland), for the production of rose oxide ${ }^{8}$. The reaction path is shown in Figure 13. 
INDUSTRIAL APPLICATIONS OF PHOTOCHEMISTRY

1. Diene-reactions<smiles>CC1=CC=C(C(C)C)CC1</smiles>

2. Ene-reactions<smiles>CC(C)=C(C)C</smiles>

<smiles>C=C(C)C(C)(C)O</smiles>

3. $(2+2)$-Cycloadditions<smiles>[R20]C=CC([R20])=C</smiles><smiles>[R20]C1OOC1[R20]</smiles>

Figure 12. Reactions of singlet oxygen.

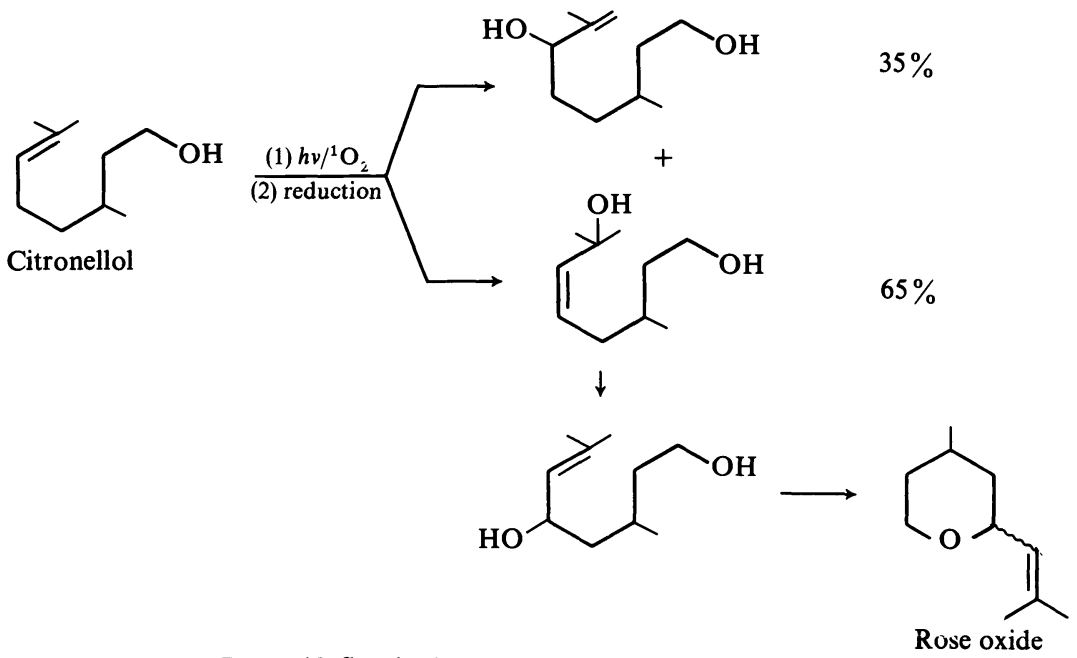

Figure 13. Synthesis of rose oxide using singlet oxygen.

A mixture of secondary and tertiary hydroperoxides is obtained by the photooxidation of citronellol in the presence of rose bengal as sensitizer. Reduction of this mixture with bisulphite yields the corresponding alcohols. Allylic rearrangement of the main product in acid solution and subsequent dehydration leads to a mixture of the stereoisomeric rose oxides, which are used as perfumes. 
These syntheses, however, by no means exhaust the technical aspects of photosensitized oxidation. Thus there is considerable interest in clarifying the part played by singlet oxygen in the photodegradation of dyes and in the protective mechanisms that nature has developed to counter undesired photooxidations. The best known is the protection of chlorophyll by $\beta$ carotene, which can deactivate singlet oxygen very effectively ${ }^{9}$.

Completely analogous to this, energy acceptors are applied technically for stabilizing plastics and fibres. Like $\beta$-carotene, they act as energy sinks.

\section{Type of reaction}

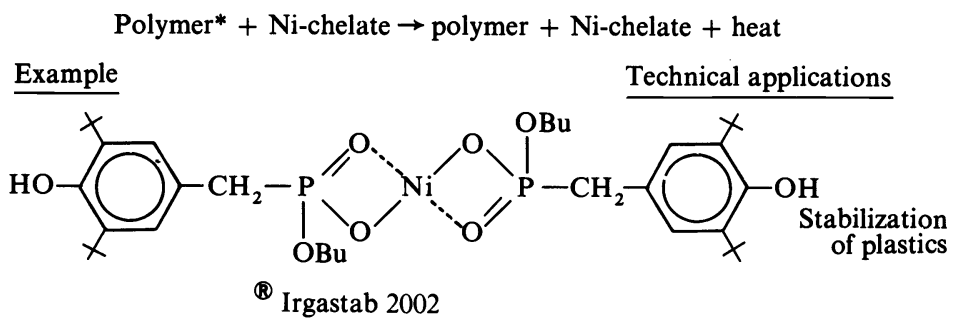

Figure 14. UV-stabilization of plastics by quenching.

Nickel chelates are used in practice for stabilizing plastics, because they can quench excited polymer molecules in both their singlet and triplet states with high efficiency ${ }^{10}$. Thus energy transfer has technical significance for both the formation and deactivation of electronically excited atoms or molecules.

\section{ISOMERIZATION}

In the last fifteen years, particularly spectacular results have been achieved by synthetic photochemistry in the field of isomerization. They have greatly enriched organic chemistry by contributing a large number of unusual compounds, some of which have structures that were formerly considered to be impossible. Unfortunately, these exotic compounds have still not acquired any commercial significance. It remains a disappointment that no clear correlation exists between the exacting chemistry, the complicated structures, and the particularly valuable technical properties. Up to now, industrial applications have been found for only two photochemical isomerization reactions: an electrocyclic ring opening in the vitamin D synthesis and a thermoreversible hydrogen shift in the ultra-violet stabilization of plastics.

The synthesis of vitamin $D_{3}$ from 7-dehydrocholesterol is very important in human medicine and also for animal nutrition. When 7-dehydrocholesterol is exposed to light, the cyclohexadiene ring is opened to a triene, viz. previtamin $D_{3}$. On gentle heating, this isomerizes to vitamin $D_{3}$ as a result of a sigmatropic 1,7-hydrogen shift. By far the largest manufacturer of vitamin $\mathrm{D}_{3}$ is Philips-Duphar in Holland (Figure 15). 

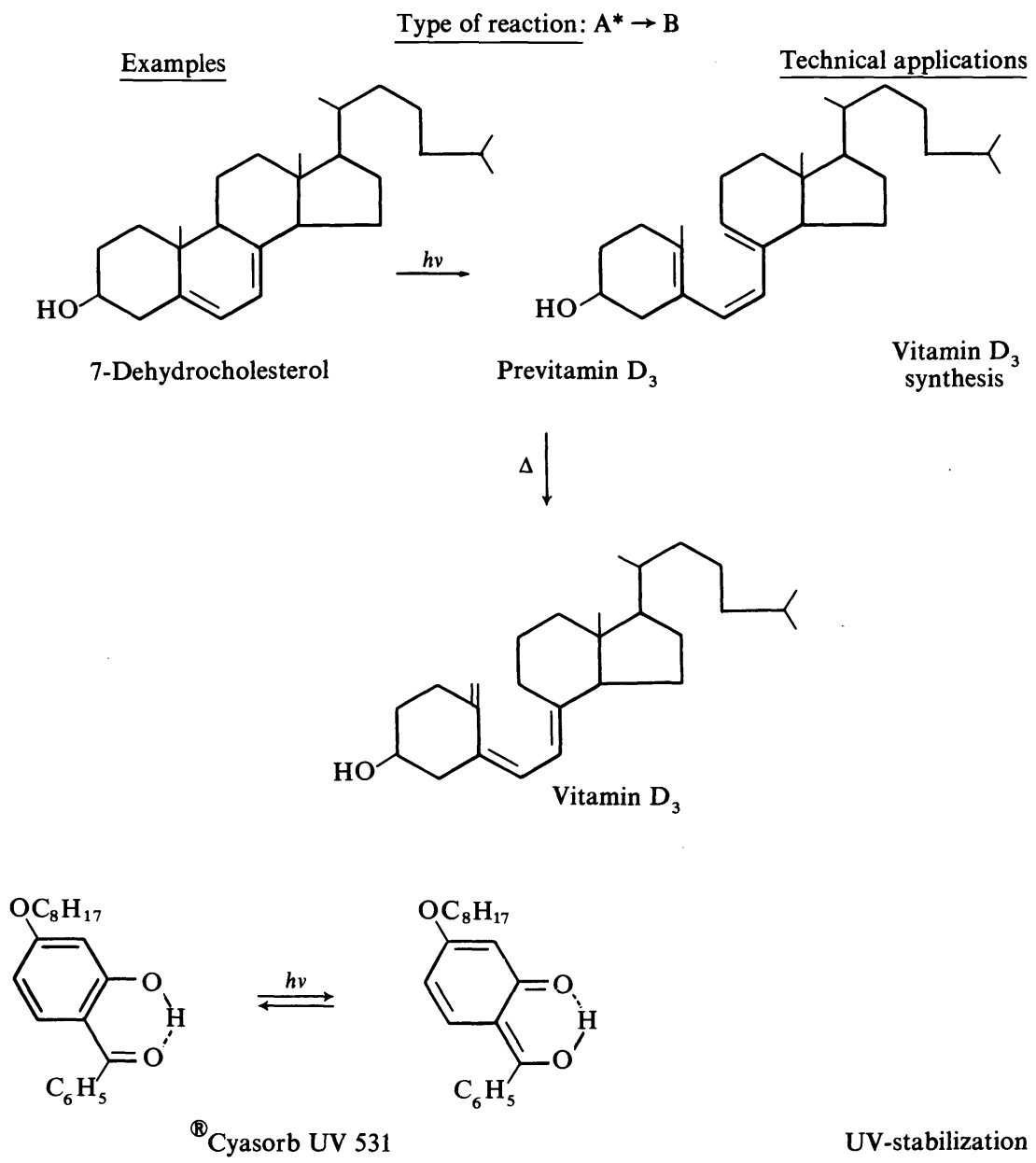

Cyasorb UV 531

UV-stabilization<smiles></smiles>

${ }^{\circledR}$ Tinuvin P

Figure 15. Isomerization.

The principle underlying the action of the main absorbers used to protect plastics from ultra-violet degradation is a thermoreversible hydrogen shift ${ }^{10}$. The best known is the stabilization mechanism of $o$-hydroxybenzophenones. When these compounds are exposed to light, photo-enols are formed by hydrogen migration, the reaction being favoured by an intramolecular hydrogen bridge. The photo-enols are very rapidly thermally re-isomerized 
back to the original ketones. It is probable that 2-(o-hydroxyphenyl)benztriazoles, which are still more important technically, convert light energy to heat by a similar mechanism.

\section{ADDITION}

The gap between the results of scientific research and their practical exploitation is even greater in the field of photoaddition than it is with photoisomerization. Thus even the elegant cycloadditions of maleic an-<smiles>O=C1C=CC(=O)O1</smiles>

Figure 16. Addition.

hydride ${ }^{11}$ to aromatics or of carbonyl compounds to olefins ${ }^{12}$ have only led to patent applications but not to any commercial products as yet. Only the cinnamic acid dimerization has found practical use in u.v.-curable coatings (Figure 16).

One reason for the poor technical exploitation of photoaddition reactions might very well be that there is still not enough cooperation between industrial chemists and photochemists. There is no doubt that compounds produced up to now by photochemical means have carried too few functional groups that may have been attractive springboards for further reactions yielding biologically active products. It is assumed that two structural elements are usually required for biological activity ${ }^{13}$. First of all, an active 


\section{INDUSTRIAL APPLICATIONS OF PHOTOCHEMISTRY}

site is needed for the actual biological effect; and, secondly, there must be a lipophilic group in the molecule to ensure transportation from the point of application to the intended organ. It is precisely for the carrier function that photoaddition products, which often have unusual structures, appear to be interesting for facilitating penetration of cell membranes.

\section{HYDROGEN ABSTRACTION}

The problem of thinking out applications for a photochemical reaction is greatly simplified if radicals are involved. In this case, photochemistry can be tied in with the wide and technically important field of radical chemistry. An example of this is the well-known hydrogen abstraction.

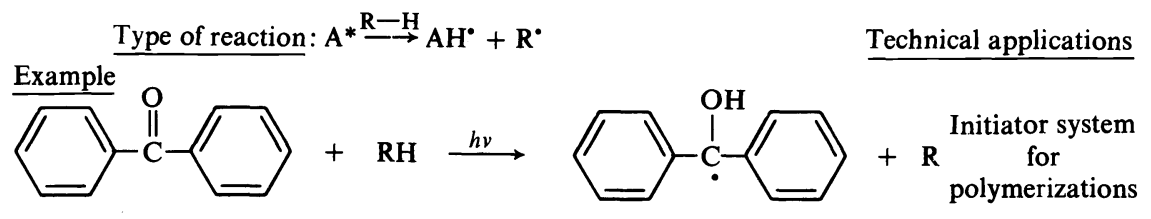

Figure 17. Hydrogen abstraction.

The abstraction of hydrogen is a frequently encountered reaction of ( $\mathrm{n}, \pi^{*}$ )-excited carbonyl compounds. It has been most thoroughly investigated in connection with the photoreduction of benzophenone to benzopinacol. The intermediately formed radicals are suitable for triggering off polymerization and crosslinkage reactions. A drawback of this system, however, is that benzophenone is practically ineffective in precisely those common polymerization systems that contain styrene, even if there are groups present that are suitable for hydrogen abstraction. In these solutions, styrene $\left(E_{\mathrm{T}}=61 \mathrm{kcal} / \mathrm{mol}\right)$ quenches the triplet excited benzophenone $\left(E_{\mathrm{T}}=68.5\right.$ $\mathrm{kcal} / \mathrm{mol}$ ) and polymerization does not take place $^{14}$.

\section{FRAGMENTATION}

Photochemically induced fragmentation is technically a more important method of producing radicals than hydrogen abstraction. Fragmentation has penetrated further into industrial chemistry than any other primary photochemical process. The applications range from initiator systems for light-curable coatings and printing inks to the commercial-scale sulphochlorination and photooximation production processes. A few important sectors will be dealt with in somewhat more detail below.

Photoinitiators are used to induce photopolymerization and photocrosslinking ${ }^{15}$. Both processes have acquired great importance in current practice and will probably become even more attractive in future. Fragmentation is the commonest primary photochemical step in photoinitiation.

The most important ingredients of a photopolymer system are:

(a) monomers that can be polymerized by the species formed during exposure

to light, and 
Type of reaction: $(\mathrm{A}-\mathrm{B})^{*} \rightarrow \mathrm{A}+\mathrm{B}$

Examples

Technical applications

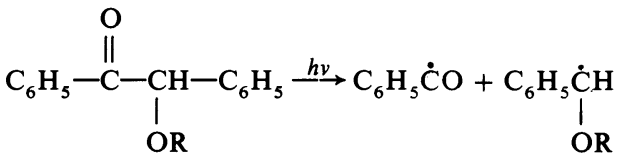

Initiator systems for:

paints and primers, light curable printing inks, photopolymer printing plates, photoresists,<smiles>[R]OS(=O)(=O)c1ccc2c(c1)C=CC(=N)C2=O</smiles><smiles>[R12]c1ccc([NH2+]Cl)cc1</smiles>

$\mathrm{Cl}_{2} \stackrel{h v}{\rightarrow} 2 \mathrm{Cl}^{-}$

$\mathrm{NOCl} \stackrel{h v}{\longrightarrow} \mathrm{NO}+\mathrm{Cl}^{\circ}$<smiles></smiles><smiles>[R12]C1=CC=[C+]C=C1</smiles>

diazotype process offset printing plates,

starters for radical chain reactions, chlorination, sulphochlorination, photooximation

Figure 18. Fragmentation.

(b) the iritiator system that is stimulated by light.

Feasible monomers for this technique are many of the common types used in the manufacture of plastics. A number of compounds that trigger off polymerization on exposure to light have been suggested as initiators. Nevertheless, comparatively few systems have managed to gain a foothold in practice, because good photoinitiators must satisfy a number of requirements.

\section{Requirements imposed on photoinitiators}

(1) They must be highly effective in as low concentrations as possible.

(2) Mixtures of photoinitiator and monomers must have high storage stability in darkness.

(3) The initiator must absorb in the $320-420 \mathrm{~nm}$ range with $\varepsilon>10^{2} 1 \cdot \mathrm{mol}^{-1}$. $\mathrm{cm}^{-1}$.

(4) The initiator must be adequately soluble in the binder and should not cause yellowing after curing.

Photopolymer systems are primarily used for u.v.-curable coatings ${ }^{16}$. These can be described in general terms as systems in which exposure to light brings about typical changes in physical properties, e.g. changes in solubility, adhesion or mechanical strength. They are mainly used in the furniture, printing and electronic industries, and in precision mechanics. 


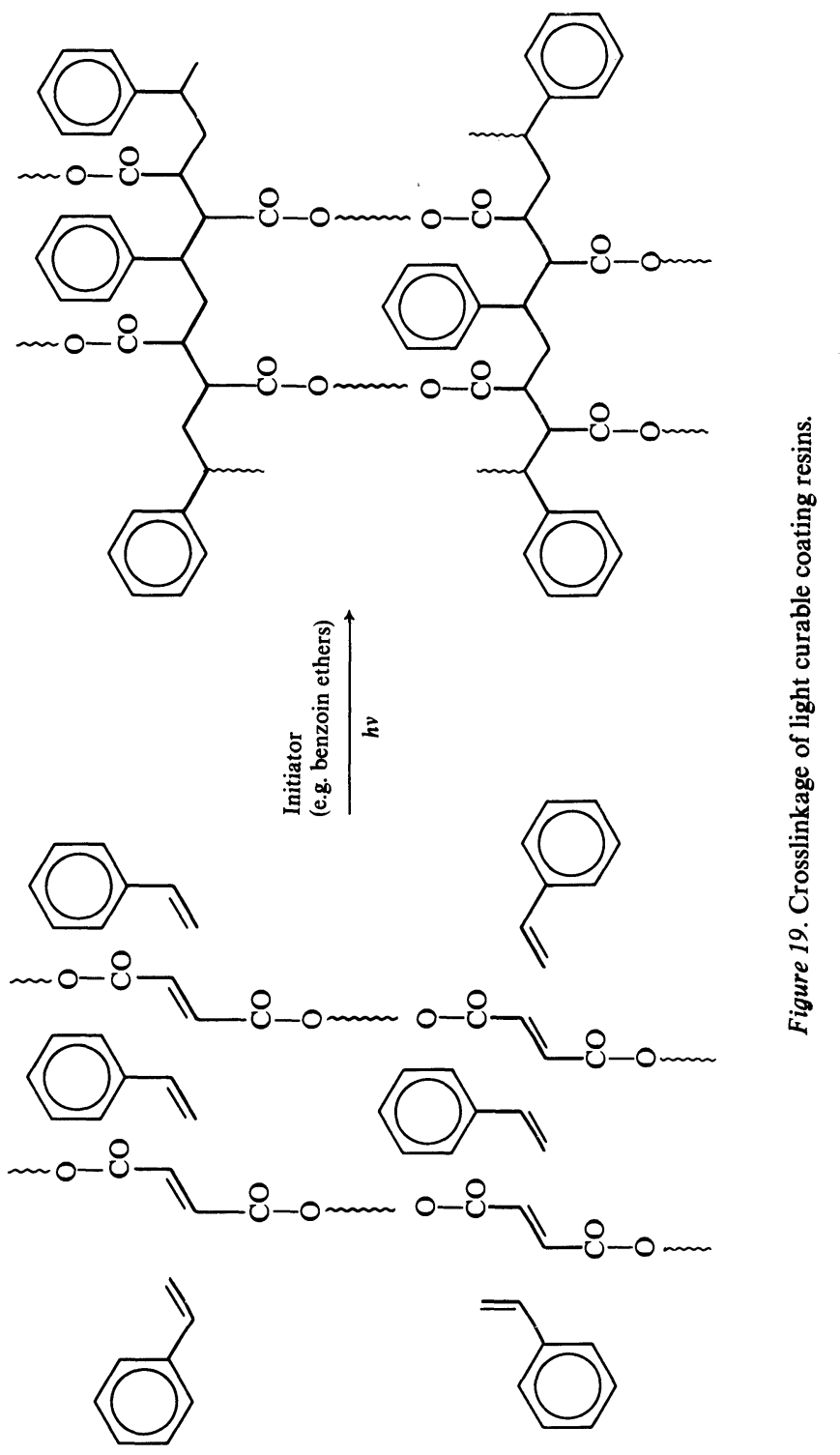


Some u.v.-curable unsaturated polyester resins have been on the market for about ten years. They are used in primers for particle board in the furniture trade.

The primers consist essentially of a solution of polyesters of fumaric acid in styrene and contain one to three per cent of a photoinitiator, e.g. a benzoin derivative. Coats of about $0.1 \mathrm{~mm}$ thickness are applied to the particle board and then exposed to high-pressure mercury-vapour lamps. The photoinitiator thus splits into radicals, which induce crosslinkage of the polyester chains as a result of copolymerization of the styrene with the double bonds in the fumaric acid. At a rating of about $10 \mathrm{~kW} / \mathrm{m}^{2}$ of exposed surface, curing is completed within a few seconds.

Light-curing of printing inks is also gradually becoming accepted in practice, because it eliminates many of the problems inherent in classical drying techniques ${ }^{17}$.

Photopolymeric printing plates and photoresists are other important, or perhaps the most important, technical fields of applications for coatings that are cured by light ${ }^{18}$.

The first commercial relief printing plates were brought on to the market in 1957 by Dupont under the name of ${ }^{\circledR}$ Dycril. Other companies followed in quick succession, e.g. Grace with ${ }^{\circledR}$ Letterflex, BASF with ${ }^{\circledR}$ Nyloprint, Asahi with ${ }^{\circledR}$ APR and many more.

In principle all these printing plates function in the same manner. Some kind of light-sensitive photopolymer system, consisting of a polymer matrix, a crosslinking agent and a photoinitiator, is applied to a carrier, e.g. a metal foil or a plastics film. This is then exposed through a pattern. The unexposed parts are washed out with a developer, and the remaining photopolymeric relief is used direct as a matrix for printing.

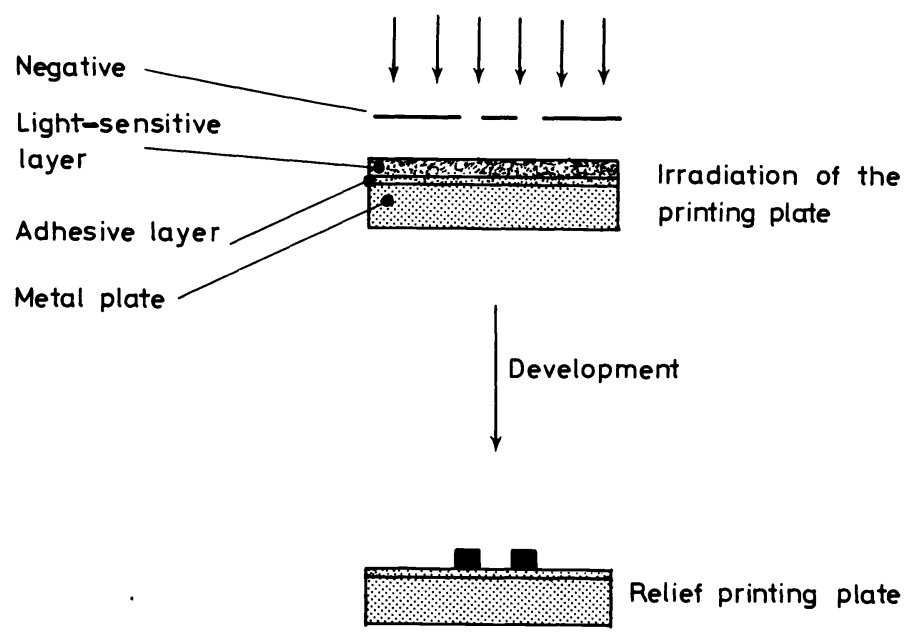

Figure 20. Structure and method of operation of a photopolymer printing plate. 


\section{INDUSTRIAL APPLICATIONS OF PHOTOCHEMISTRY}

Rapid light-curing of relatively thick layers was one of the important requirements that had to be fulfilled for the technical realization of this process. In the beginning, when this was not possible, the relief was obtained in a slightly different manner. A light-curable thin coating was applied to a zinc plate, exposed, and developed. The exposed areas remained on the plate in the form of a hardened acid-resistant image. The uncovered parts of the metal plate were etched by nitric acid until a relief was obtained suitable for printing.

Although this principle has lost ground in the graphics industry as a result of the emergence of the photopolymeric relief plates, it is indispensable in etching and electroplating. Here microelectronics have roused a demand for structural parts with dimensions of a few thousandths of a millimetre, e.g. for printed circuits, precision scales on glass or metal, precision mouldings in fine mechanics, and lens masks in kinescopes for colour television sets.

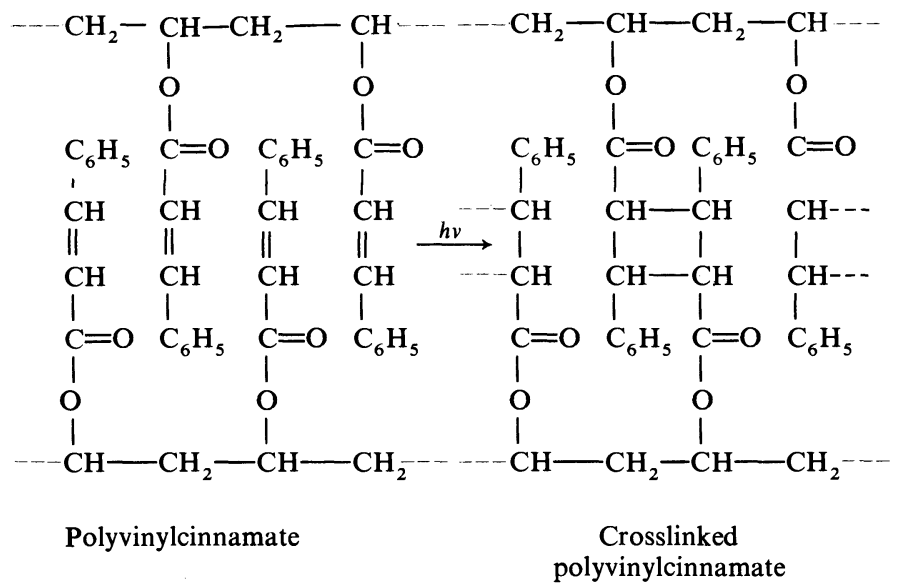

Figure 21. Curing polyvinylcinnamate by light.

The best-known system starts with polyvinyl alcohol, which is esterified with cinnamic acid. On exposure to light in the presence of sensitizers, the polyvinylcinnamate chains are crosslinked via the double bonds of the cinnamic acid. The result is a sudden decrease of the solubility in organic solvents.

Non-radical fragmentation has also gained a foothold in reproduction techniques.

On exposure to light, $o$-diazoquinones are converted into acids, an intermediate step being the formation of a ketene. This reaction represents the common basis of positive offset printing systems in which the diazoquinone is bonded to a lipophilic polymer via the residual R-group. The photosensitive polymers thus obtained become soluble in alkali when they are exposed to light (Figure 22). 


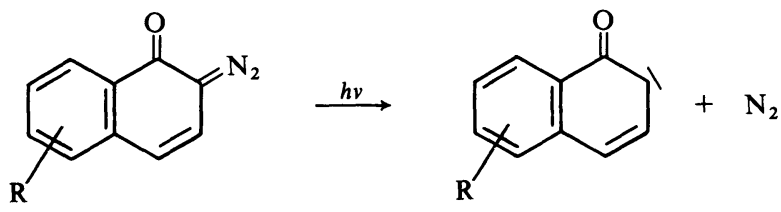

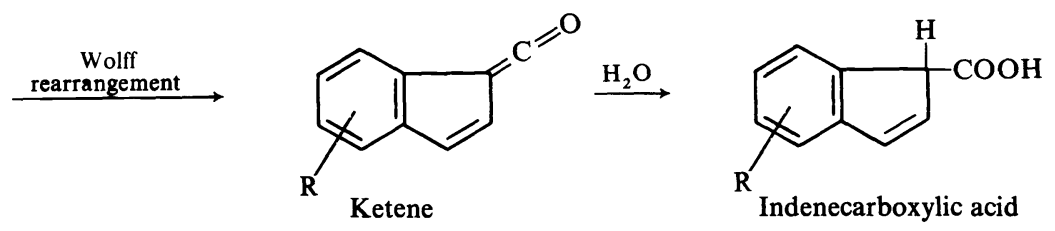

Figure 22. Photolysis of $o$-diazoquinones.

Presensitized printing foils for offset printing ${ }^{19}$ consist of a carrier, e.g. aluminium, covered with a photosensitive coating of about $2 \mu \mathrm{m}$ thickness. After this coating has been exposed under a negative, its solubility in certain solvents changes rapidly. The term positive or negative system is adopted according to whether the exposed part of the coating is soluble or insoluble in the developer. The image thus formed on the surface of the carrier consists of hydrophobic zones, which take up the oily printing ink, and hydrophilic zones, which repel the ink. Diazonium salts that become insoluble in water on exposure to light have been widely accepted for negative systems (Figure 23).

- Nowadays, offset printing accounts for about 25 per cent of all printed matter. One quarter of all American newspapers, i.e. about 15 million copies a day, are printed by offset techniques.

In general, the main advantages of photomechanical printing over labourintensive techniques with lead types for the characters and etched zinc plates

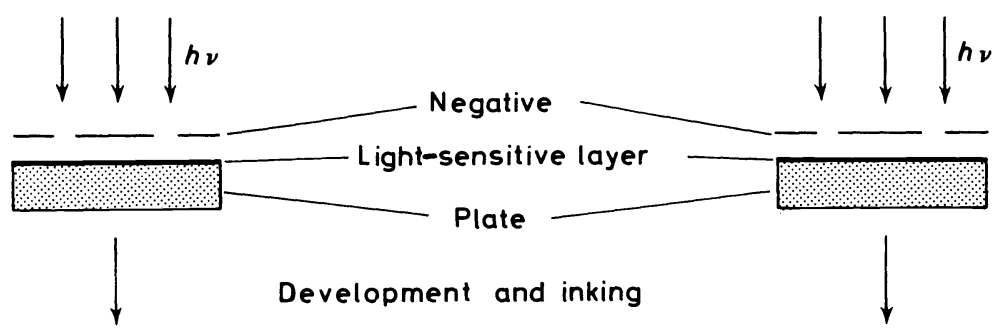

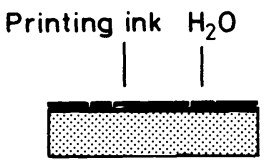

Positive system

( 0 - diazoquinones)

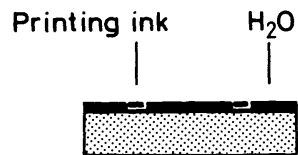

Negative system

(diazonium salts)

Figure 23. Structure and mode of operation of positive and negative offset printing plates. 
for the illustrations are:

(1) Photosetting may be adopted, because a film is the information carrier for all motifs.

(2) Photopolymer printing plates are considerably lighter than the classical lead blocks. This fact gives rise to simpler printing presses running at higher speeds.

(3) The plates are flexible and can be bent for rotary printing.

Reproduction techniques embrace copying as well as printing. The ${ }^{\circledR}$ Ozalid process for diazotypes is well known, although it has lost much of its former importance due to the process of electrophotography mentioned before.

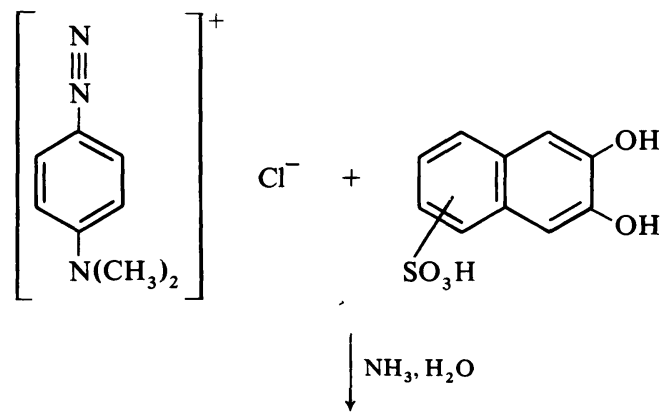<smiles>Cc1ccc2cc(O)c(O)c(N=Nc3ccc(N(C)C)cc3)c2c1</smiles>

Figure 24. Diazotype process $\left({ }^{\circledR} \mathrm{Ozalid}\right.$ process, Kalle).

A diazotype is produced by a coupling reaction between a diazonium salt and a phenol. The photosensitive layer contains a diazonium salt, a phenol and an acid that prevents premature coupling. When the copying foil is exposed through the original, the diazonium salt under the transparent parts is decomposed. Moist ammonia gas is then passed over the foil to neutralize the acid. As a result, the remaining diazonium salt is free to react with the coupling agent, and the desired type character appears in colour.

Photochemical initiation of radical chain reactions also plays a major part in the technical syntheses of compounds with low molecular weights. 


\section{MARTIN PAPE}

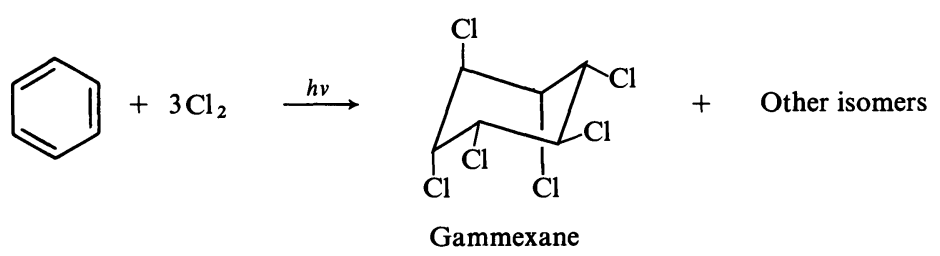

Figure 25. Photochlorination of benzene.

The greatest economic significance in photochlorination is attached to the synthesis of $\gamma$-hexachlorocyclohexane (gamma-BHC or lindane). In this case, the photochemical process had already ousted thermal chlorination some twenty years ago, because it can be carried out at lower temperatures thus allowing higher yields of the desired $\gamma$-isomer ${ }^{20}$. Even today, this product is still an important insecticide and rodenticide. World sales in 1973 were about $\$ 70$ million.

$$
\begin{aligned}
& \mathrm{RH}+\mathrm{SO}_{2}+\mathrm{Cl}_{2} \stackrel{h v}{\longrightarrow} \mathrm{R}-\mathrm{SO}_{2} \mathrm{Cl}+\mathrm{HCl} \\
& \mathrm{RH}+\mathrm{SO}_{2}+\frac{1}{2} \mathrm{O}_{2} \stackrel{h v}{\longrightarrow} \mathrm{R}-\mathrm{SO}_{3} \mathrm{H}
\end{aligned}
$$

Figure 26. Phosulphochlorination and photosulphoxidation.

The Reed sulphochlorination is mainly resorted to for the manufacture of surfactants from paraffins. It also plays a part in the modification of polyethylene in special grades of rubber, such as Dupont's ${ }^{\circledR}$ Hypalon. The sulphoxidation of paraffins has profited from the trend towards readily degradable detergents. Thus Farbwerke Hoechst have a plant on stream that produces about 12000 tons of sulphonates a year by this process.

The economic success of photoinduced radical chain reactions with quantum yields of up to $10^{3}$ under technical conditions, appeared to confirm for a long time the prejudice that photoreactions with stoichiometrical light consumption were much too expensive for commercial production and could only be considered for specialities with a high price, such as vitamin D. For this reason, I regard the technical realization of photooximation as the most important event of the last ten years in industrial photochemistry ${ }^{21}$. In view of its general significance, I shall deal with this process in more detail.<smiles></smiles>

Figure 27. Photooximation of saturated hydrocarbons.

Photooximation of saturated hydrocarbons was discovered in 1919 by E. V. Lynn ${ }^{22}$. By a coincidence, this American chemist let a solution of nitrosyl chloride in $n$-heptane, which he had prepared for quite a different 
purpose, stand for a day in the sunlight. The originally red-brown solution turned blue and was then completely discoloured. A heavy, yellow oil had collected at the bottom of the vessel, and Lynn identified it as a mixture of the hydrochlorides of the isomeric heptanone oximes.

This first photooximation experiment already demonstrated the ease with which oximes could be produced in one step under such mild conditions from hydrocarbons that were considered to be inert. Nevertheless, practical interest in photooximation was not aroused until twenty to thirty years later, when the hectic growth of polyamide fibres caused a worldwide search for cheaper syntheses for the starting products.



Figure 28. Caprolactam via photooximation.

The application of photooximation to cyclohexane opened up the shortest route to caprolactam and Nylon 6. Today, Toyo Rayon in Japan operates the process on a 150000 tons-a-year scale. Thus the risk has been taken of launching a commercial scale photoreaction process with a quantum yield of less than one for a product that sells at about $\$ 0.5 / 1 \mathrm{~b}$ on the world market and for which there is already an annual production capacity of about 1.8 million tons by other processes. Since then, Aquitaine-Organico have taken a plant on stream for producing by photooximation 4000 tons of lauryl lactam a year for Nylon 12.
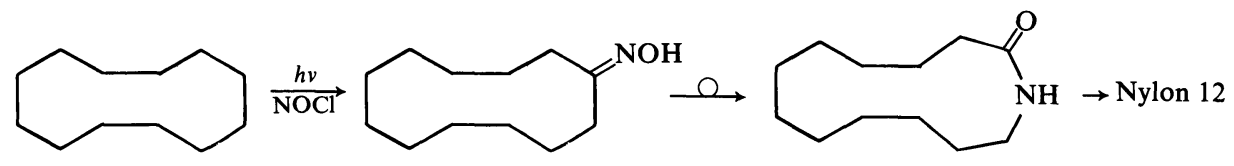

Figure 29. Lauryl lactam by photooximation.

Nylon 12 has found a number of important special applications, because it is the polyamide that has the lowest density and absorbs the least moisture. So it is used for dimensionally stable plastics articles, foodstuffs packaging, and plastics coatings on metals.

These encouraging examples demonstrate that we are now technologically in a position of carrying out photoreactions so cheaply that they can compete with conventional processes even on a commercial scale. Thus the industrial chemist has much better prospects than before of economically exploiting the results of photochemical research. Those aspects which are most likely to arouse an industrial chemist's interest when reading a scientific publication on photochemistry are listed hereunder: 


\section{Criteria for photochemical papers of technical interest}

The photoreaction described:

leads to products that can be commercialized, can be adopted for compounds that are readily available,

is superior to conventional methods,

yields reactive intermediates,

proceeds with high selectivity and quantum yield.

Lynn's publication in 1919 on photooximation satisfied almost all these conditions, and perhaps there are still more cases in the literature that we have not yet been lucky enough to find. But even if only a few of these conditions can be met, it is frequently worthwhile studying the practical feasibility of a photoreaction more closely.

This ends my review. Its aim was to demonstrate that applied photochemistry is growing up and is seeking to strengthen the ties with pure photochemistry in universities.

\section{ACKNOWLEDGEMENTS}

The chemists of our Organic Photochemistry Group in the BASF Main Research Laboratory substantially assisted me with their knowledge and advice in compiling this review. I should like to thank the head of the group, Dr Martin Fischer, and his co-workers Dr Eckhard Hickmann, Dr Günter Vitt and Dr Werner Küsters.

\section{REFERENCES}

1 M. Clausen, Chemie in unserer Zeit, 7, 141 (1973).

2 American Cyanamid Corp., US Pat. Nos. 3576987 (1967) and 3597362 (1965);

K.-D. Gundermann, Fortschr. chem. Forsch. 46, 61 (1974).

${ }^{3}$ H. Janistyn, Handbuch der Kosmetika und Riechstoffe, Vol. III, p 708. Alfred Hüthig Verlag (1973).

4 V. Tulagin, J. Opt. Soc. Amer. 59, 328 (1969).

${ }^{5}$ Data provided by Quarzlampen Gesellschaft, Hanau (1974).

6 Toyo Rayon, Deutsche Auslegeschrift Nr.1493257 (1965).

7 G. O. Schenck and K. Ziegler, Naturwissenschaften, 32, 157 (1944).

8 F. Ohloff, E. Klein and G. O. Schenck, Angew. Chem. 73, 578 (1961).

9 C. S. Foote, Y. C. Chang and R. W. Denny, J. Amer. Chem. Soc. 90, 5216 (1970).

O. Cicchetti, Advanc. Polymer Sci. 7, 70 (1970).

11 R. Steinmetz, Fortschr. chem. Forsch. 7, 445 (1967).

2 D. R. Arnold, Advanc. Photochem. 6, 301 (1968).

13 A. Leo, C. Hansch and D. Elkins, Chem. Rev. 71, 525 (1971).

14 H.-G. Heine, H.-J. Rosenkranz and H. Rudolph, Angew. Chem. 84, 1032 (1972).

15 G. Oster and N. Yang, Chem. Rev. 68, 125 (1968).

16 G. A. Delzenne, J. Macromol. Sci.-Revs. Polymer Technol. D1 (2), 185 (1971);

J. L. R. Williams, Fortschr. chem. Forsch. 13, 227 (1969/70).

17 A. Bassl and K.-H. Böhm-Kasper, Farbe und Lack, 13, 916 (1967).

18 T. Warashina and T. Kai, Japan Plastics Age, 19 (1972).

19 O. Süs,.J. Munder and H. Steppan, Angew. Chem. 74, 985 (1962).

20 L. J. Governale and J. T. Clarke, Chem. Engng Progr. 52, 281 (1956).

21 Y. U. Ito, Kogyo Kagaku Zasshi, 72,77 (1969).

22 E. V. Lynn, J. Amer. Chem. Soc. 41, 368 (1919). 